\title{
Age associated fluoride induced ultra structural and biochemical changes in rat brain
}

\begin{abstract}
Fluoride $(\mathrm{F})$ ingestion is well known to be associated with oxidative stress in experimental animals, but the precise mechanism of its toxicity associated with advancing age has not yet been delineate. The present study attempts to assess the learning and memory capacity of rats using water maze test for cognitive functioning. The markers of oxidative stress i.e., Reacting oxygen species (ROS), protein carbonyl content (PC), lipid peroxide levels (LPx), superoxide dismutase (SOD), catalase (CAT), glutathione peroxidase (GPx) and reduced glutathione (GSH) were measured in the brain hippocampus of young and aged rats fed with fluoride $(100 \mathrm{ppm})$ with drinking for 90 days. We observed significant changes of fluoride treated young and aged rat as compared with their respective controls. Lipofuscin content was significantly increased in fluoride treated aged rats. Ultra structural studies of the CA1 region in hippocampus of fluoride exposed rats revealed that the changes were more pronounced in the aged treated rats in terms of presence of clustered lipofuscin, vacuolization, lysosomal degradation and nucleolar fragmentation. On the basis of the results it may conclude that fluoride may be linked with neuro lipofuscinogenesis and alteration in neurobehavioral activity and these changes may be responsible for the development of age related disorders.
\end{abstract}

Volume I Issue 5 - 2015

\author{
Sandeep Tripathi, Dushyant Singh Chauhan, \\ Vivek Pratap Singh, Devesh Kumar Joshi, \\ Manisha Choudhary \\ National Referral Centre for fluoride poisoning in India, \\ Institute of Advanced Sciences \& Technology, NIMS University, \\ India
}

Correspondence: Sandeep Tripathi, Assistant Professor of Biotechnology, Head (R \& D) National Referral Centre for Fluoride Poisoning in India, NIMS University, India, Tel +918955577013, Email sandeeptripathiphd@gmail.com

Received: March 19, 20I5 | Published: December 07, 2015

Keywords: fluoride, aging, brain, oxidative stress

Abbreviations: ANOVA, analysis of variance; CAT, catalase; GSH, glutathione; GSSG, oxidized glutathione; GPx, glutathione peroxidase; GR, glutathione reductase; LPO, lipid peroxidation; lipofuscin (LIF); ROS, reactive oxygen species; SOD, superoxide dismutase; PMS, phenazinemethosulphate; NBT, nitoblu tetrazolium; YC, young control; YEx, young experimental; OC, old control; OEx, old experimental

\section{Introduction}

Fluorine is abundantly found in the ground drinking water in worldwide and very little amount is required to humans. Fluorosis is one of the manifestations of chronic poisoning from long-term exposure to high levels of fluoride, and is a serious health problem in many parts of the world where drinking water contains more than 1-1.5-ppm of fluoride. ${ }^{1}$ There have been numerous researches on the harmful effects of fluoride intake in excess on bones and teeth of human as well as animal models. ${ }^{2}$ It is characterized by clinical manifestations in bones and teeth. Fluoride accumulation in brain was observed in chronic high-fluoride exposure through drinking water. It is reported that high fluoride is known to cause structural and functional changes in the brain of experimental animals. ${ }^{3}$ Evidence from clinical and animal model studies demonstrates that fluoride content may increases with age. It may be either due to increased exposure with age or decreased ability to remove fluoride from the body. Therefore, knowledge of age related effects of fluoride in tissues and relative risk as well as extent of its retention in tissue is important for the understanding of its effects and associated disorders. ${ }^{4}$ The possible mechanism of fluoride induced neurotoxicity has been related to cell damage via free radical production. ${ }^{5}$ Increased lipid peroxidation (LPO) is one of the major consequences of oxidative stress. ${ }^{6}$ Lipofuscin, a morphological biomarker of lipid peroxidation, represents an end - product of oxidative degradation of lipids by free radical mechanism. ${ }^{7}$ Studies on age associated and fluoride mediated toxic effects are very important as there are several reports that, on one hand the efficiency of the blood brain barrier (BBB) may decline with advancing age, ${ }^{8}$ and on the other hand, cognitive decline may account for functional and morphological changes in the central nervous system (CNS). There is, however, a paucity of studies on the effect of fluoride intoxication with advancing age on oxidative stress.

In view of the aforementioned considerations, the present study was designed to assess fluoride induced neurochemical and ultra structural changes in the hippocampus of young and old rat brain. To achieve these objectives we administered sodium fluoride (100ppm) through drinking water to young and aged rats for 90 days. Thereafter, the animals were sacrificed and neurochemical and ultra structural studies were carried out. In the present study, we attempt to assess the effect of intake of high-fluoride levels in drinking water during fetal, weanling, and post-weaning stages of life on oxidative stress in the brain of rats.

\section{Materials and methods}

\section{Animals}

Forty male Rattus norigious, Wistar strain rats of two age groups, young ( 6 month, weight $182 \pm 2.2 \mathrm{~g}, \mathrm{n}=24$ ) and old ( 24 month, weight $466 \pm 2.5 \mathrm{~g}, \mathrm{n}=24)$, were taken from animal house of the NIMS University, Jaipur, India. The animals were separately housed in polypropylene cages in a room, which was maintained at a temperature of $22 \pm 2^{\circ} \mathrm{C}$, relative humidity of $50 \pm 10 \%$ and $12 \mathrm{~h}$ light dark cycles. They were fed a commercial pellet diet and allowed access to water ad libitum. The Institutional Animal Ethics Committee approved the study prior to the initiation of the experiment and also approved all experimental protocols. 


\section{Treatment}

Groups of young $(n=20)$ and aged $(n=20)$ animals were randomly divided into two subgroups $(\mathrm{n}=10)$ viz. young control $(\mathrm{YC})$, Young Experimental (YEx) and Old control (OC) and old experimental (OEx). 100ppm fluoride was given through drinking water to experimental groups of young and old rats for 90 days.

\section{Tissue homogenate preparation}

After 90 days of $\mathrm{Al}$ administration rats were sacrificed their brain were removed and weighed individually. Thereafter, hippocampus was dissected out for biochemical analysis. Ten percent (w/v) homogenate of the frontal cortex was prepared by of York's homogenizer fitted with Teflon plunger in $0.1 \mathrm{M}$ phosphate buffer $(\mathrm{pH}$ 7.1). The whole homogenate was first centrifuged at $2500 \mathrm{xg}$ for 10 minutes in a refrigerated centrifuge. The pellet consisting of nuclear fraction and cell debris was discarded. The supernatant was further centrifuged at $11,000 \mathrm{xg}$ for 15 minutes and mitochondrial fraction was separated. The clear supernatant was further centrifuged at $105,000 \mathrm{xg}$ for 90 minutes and the resultant supernatant was used for determining enzyme activities.

\section{Total reacting oxygen species (ROS) assay}

The basal level of ROS was determined by the procedure of Montoliu et al. ${ }^{9}$ An appropriate volume of freshly prepared tissue homogenate was diluted in $100 \mathrm{~m} M$ potassium phosphate buffer $(\mathrm{pH} 7.4)$ and incubated with a final concentration of $5 \mathrm{~m} M$ dichlorofluorescin diacetate in methanol for $15 \mathrm{~min}$ at $37^{\circ} \mathrm{C}$. The dyeloaded samples were centrifuged at $12,500 \mathrm{~g}$ for $10 \mathrm{~min}$ at $4^{\circ} \mathrm{C}$. The pellet was vortex mixed at ice-cold temperatures in $5 \mathrm{ml}$ of $100 \mathrm{M}$ phosphate buffer $(\mathrm{pH} 7.4)$ and again incubated for $60 \mathrm{~min}$ at $37^{\circ} \mathrm{C}$. The fluorescence measurements were performed with a Hitachi 850 spectrofluorometer at $488 \mathrm{~nm}$ for excitation and $525 \mathrm{~nm}$ for emission wavelengths. The cuvette holder was maintained at $37^{\circ} \mathrm{C}$. ROS were quantified from the dichlorofluorescein standard curve in methanol $(0-100 \mathrm{n} M)$.

\section{Measurement of protein oxidation and lipid peroxidation}

The protein content was measured by the method of Lowry et al. ${ }^{10}$ using bovine serum albumin (BSA) as standard. The protein oxidation was measured by estimating the protein carbonyl levels by the method of Liu et al. ${ }^{11}$ Protein carbonyl content was determined in the samples by measuring the DNPH adducts at $375 \mathrm{~nm}$. Carbonyl contents were calculated by using a molar extinction coefficient $(e)$ of $22,000 \mathrm{M}^{-1} \mathrm{~cm}^{-1}$. Data were expressed as nmoles carbonyl $/ \mathrm{mg}$.

\section{Lipid oxidation and lipofuscin}

The lipid peroxide (LPx) levels were measured by the method of Ohkawa et al. ${ }^{12}$ The thiobarbituric acid reacting substances (TBARS) of the sample were estimated spectrophotometrically at 532nm and expressed as nmole of MDA /g tissue. Lipid hydroperoxide ( $\mathrm{LOOH})$ levels were measured by the method of Haldebrandt. ${ }^{13}$ In this method, ferrousthiocynate was measured on peroxidation of $\mathrm{Fe}^{2+}$ to $\mathrm{Fe}^{3+}$ by $\mathrm{H}_{2} \mathrm{O}_{2}$ and resulted in intense pale colour, which was read at $480 \mathrm{~nm}$. Standard cumine hydroperoxide was used as control and results were expressed as nmole of cumin hydroperoxide per gram/tissue. Conjugated dienes (CD) was measured by the method of Racknagel. ${ }^{14}$ The cytosolic fraction was extracted from long chain fatty acids by chloroform: methanol. The absorbance of lipid extract was read at $220 \mathrm{~nm}$ on spectrophotometer. The level was expressed as $\mu$ mole dienes / 100g lipid using the molar extinction coefficient of $2.1 \times 10^{3}$. The lipofuscin (LF) was measured according to the method of Sohal. ${ }^{15}$ Brain tissue was homogenized with 2:1 chloroform-methanol mixture. The mixture was centrifuged at 3000rpm and the pellet discarded. The concentration was measured with a fluoro-spectrophotometer at an excitation maximum of $360 \mathrm{~nm}$ and emission maximum of $420 \mathrm{~nm}$. The content of the fluorescence was determined using quinine fluorescence as a standard. Data were presented as U/g tissue. One unit $(\mathrm{U})$ of lipofuscin is defined as fluorescence of $0.01 \mathrm{~g} / \mathrm{ml}$ quinine sulphate.

\section{Measurement of endogenous enzymes}

An aliquot of cerebellum homogenate was used for the assay of enzymatic antioxidants. The superoxide dismutase (SOD EC 1: 15.1.1) activity was determined from its ability to inhibit the reduction of NBT in presence of PMS. ${ }^{16}$ The reaction was monitored spectrophotometrically at $560 \mathrm{~nm}$. The SOD activity was expressed as $\mathrm{U} / \mathrm{mg}$ protein (1unit is the amount of enzyme that inhibit the reduction of NBT by one half in above reaction mixture). Catalase (CAT, EC 1.11.1.6) activity was assayed using hydrogen peroxide as substrate; the decomposition of $\mathrm{H}_{2} \mathrm{O}_{2}$ was followed at $240 \mathrm{~nm}$ on spectrophotometer. ${ }^{17}$ The CAT activity was expressed as $\mathrm{U} / \mathrm{mg}$ protein. The glutathione peroxidase (GSHPx, EC 1.11.1.0) was assayed using GSH, NADPH and $\mathrm{H}_{2} \mathrm{O}_{2}$ as reactants. The oxidation of GSH into GSSG was measured in terms of oxidation of NADPH to NADP ${ }^{+}$and assayed as decrease in the absorbance of reaction mixture at $340 \mathrm{~nm}$ on spectrophotometer. ${ }^{18}$ The activity of GSHPx was expressed as n moles of NADPH oxidized / $\mathrm{min} / \mathrm{mg}$ protein. Glutathione reductase (EC.1.6.4.2, GR) activity was assayed by the method of Hazelton. ${ }^{19}$ Activity of GR was expressed as nmoles of NADPH oxidised $/ \mathrm{min} / \mathrm{mg}$ protein of cell extract.

\section{Measurement of reduced and oxidized glutathione}

Reduced glutathione was measured in deproteinized supernatent of the cerebellum. Tissue homogenate was deproteinated with tetrachloroacetic acid, centrifuged and supernatant was used for the estimation of reduced glutathione (GSH) with the help of Ellman reagent (5, 5' dithiobis (2-nitro benzoic acid)). The optical density of the pale colour was measured on the spectrophotometer on $412 \mathrm{~nm}$. An appropriate standard (pure GSH) was run simultaneously. The level of GSH was expressed as $\mu \mathrm{g} / \mathrm{g}$ tissue. ${ }^{20}$ The oxidized glutathione (GSSG) was estimated by the decrement of GSSG in the presence of NADPH and glutathione reductase and determined the decrement of NADPH absorbance at $340 \mathrm{~nm}$. The result was expressed as $\mu \mathrm{g} / \mathrm{g}$ tissue. ${ }^{21}$

\section{Electron microscopic study}

Ultra structural changes in frontal cortex region of the central nervous system were assessed using standard electron microscopic technique. The rats were anaesthesized with Nembutol (sodium pentobarbitol) solution, $50 \mathrm{mg} / \mathrm{kg}$ b.w. and perfused through heart with Karnovsky's fixative $(0.1 \mathrm{M}$ paraformaldehyde and glutaraldehyde solution in cocodylate buffer, $\mathrm{pH}$ 7.3). The brains were quickly removed from the cranium, placed on ice and frontal cortex was dissected out. Small pieces of $2-3 \mathrm{~mm}$ size were immersed in the same fixative for four hours, and thereafter washed with $0.1 \mathrm{M}$ cacodylate buffer ( $\mathrm{pH} 7.3)$. The samples were post fixed for three hours at $4^{\circ} \mathrm{C}$ in $1 \%$ osmium tertraoxide prepared in $0.1 \mathrm{M}$ cacodylate buffer. The 
specimen were washed with distilled water and left in $1 \%$ aqueous uranyl acetate overnight. Subsequently, dehydration was carried out in ascending grades of alcohol, acetone and in pure acetone. Following dehydration, the specimens were embedded in Epon 812 at room temperature. Sections were cut on an LKB-Ultramicrotome with a glass knife. Thereafter, sections were mounted on 300 mesh copper grids, stained with $1 \%$ uranyl acetate and lead citrate and examined with a Phillips (FEI Tecnai 12 twin) Transmission Electron Microscope.

\section{Statistical analysis}

Experimental data were summarized as Mean \pm SE ( $n=6)$. Groups were compared together by one way analysis of variance followed by Student Newman-Keuls post hoc test. The acceptance level of significance was $p<0.05$. InStat (version 3 ) was used for analysis of data.

\section{Results and discussion}

In the present study we report that exposure with fluoride caused significant oxidative degradation of lipids and proteins due to increased rate of ROS following structural changes in the hippocampus and these effects were more pronounced in aged rats. The fluoride exposed rat revealed reduced body and brain weight (Table 1). These changes seem to be linked with chronic fluoride neurotoxicity. Moreover, as these changes were more apparent in the aged rats, it is likely that they may be due to the perturbation in cellular and molecular metabolism of aged rats.

Table I Body and brain weight of control and experimental rats

\begin{tabular}{lll}
\hline Groups & Terminal body wt $(\mathrm{g})$ & Brain $(\mathrm{g} / \mathrm{kg})$ \\
\hline YC & $278.8 \pm 6.3$ & $3.9 \pm 0.07$ \\
YEx & $247.5 \pm 5.2^{\mathrm{a}}$ & $3.34 \pm 0.05^{\mathrm{a}}$ \\
OC & $534.2 \pm 8.2^{\mathrm{ab}}$ & $3.62 \pm 0.07$ \\
OEx & $422.5 \pm 9.1^{\mathrm{abc}}$ & $2.89 \pm 0.08^{\mathrm{c}}$
\end{tabular}

The body and brain weight of control and experimental rats of young and old rats are presented as Mean \pm SEM in six rat of each group. Superscripts relate significant $(p<0.05)$ comparison with YC (a), YEx (b) and OC (c).

Although toxic effects of fluoride are well established but its mechanism of action is poorly understood. Following oral administration, retention of fluoride is reported in the brain, bone, kidney, muscle and heart. ${ }^{22}$ Figure 1 embodies ROS, protein and lipid peroxidation products. The level of ROS was found to be significantly increased by $29 \%$ in fluoride treated young rats and $31 \%$ in old rats when compared with their respective controls. The ROS was $35 \%$ was found to increase in fluoride treated aged rats as comparison with fluoride treated young rats. The PC content was found to be increased by $44 \%$ in YEx and $70 \%$ OEx as compared with YC and OC respectively. Moreover, PC increased by $62 \%$ in OEx as compared to YEx. LOOH were found to be significantly elevated by $64 \%$ and $99 \%$ in fluoride treated YEx and OEx as compared to their age matched control rats. The OEx exhibited $86 \%$ elevation of $\mathrm{LOOH}$ as compared with the YEx. CD was also markedly increased by $63 \%$ in YEx and $60 \%$ in OEx as compared to their respective controls While, it was elevated by $75 \%$ in OEx when compared with YEx. The concentration of MDA was found to be significantly increased by $67 \%$ in YEx and $62 \%$ in OEx when compared with their age matched control rats. The
OEx showed 44\% greater lipid peroxidation as compared with the YEx. The concentration of LIF was found to be markedly increased by $180 \%$ in YEx and 166\% in OEx than that of YC and OC respectively. In addition, lipofuscinogenesis was significantly increased by $201 \%$ in OEx as compared with YEx.

Our results demonstrated oxidative damage of lipids, as measured by the increased levels of lipid peroxidation products (MDA, $\mathrm{LOOH}$ and $\mathrm{CD}$ ) in the hippocampus of young and aged rats following fluoride exposure (Figure 1). This may be due to increased rate of ROS production in the brain. Moreover, as the brain contains large amount of lipids that are rich in polyunsaturated fatty acids, they can readily react with free radicals and undergo peroxidation. ${ }^{23}$ These free radicals attack lipids and proteins to produce lipofuscin pigment deposition of which was revealed by our electron microscopic studies of fluoride treated rats. We also observed that the deposition of lipofuscin was accompanied by increased levels of lipid peroxide and protein carbonyl content in the hippocampal neuron of the young and aged rats following fluoride exposure.

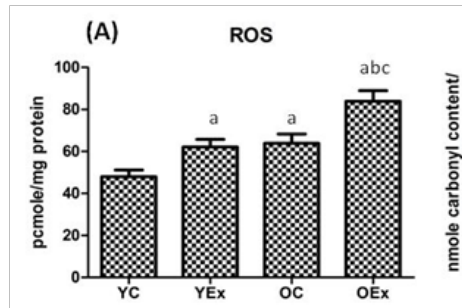

(B) $\quad P C$
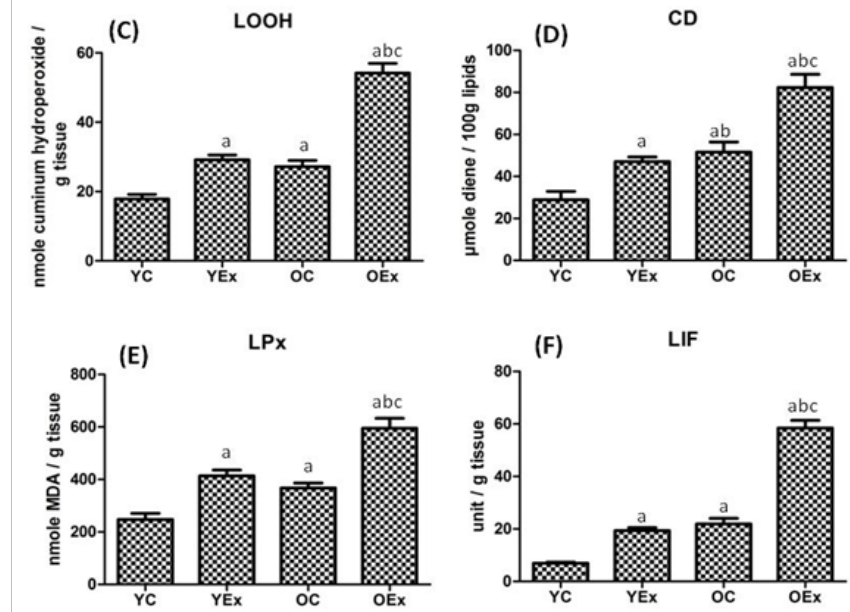

Figure I Levels of reacting oxygen species (ROS), Protein, Protein carbonyl content (PC), lipid peroxide levels (LPO) and lipofuscin (LIF) in control and fluoride treated group. The results are expressed as Mean \pm SEM in six rat of each group. Superscripts relate significant $(p<0.05)$ comparison with $Y C$ (a), YEX (b) and OC (c).

The activities of antioxidant enzymes namely SOD, CAT, GPx and GR presented in Figure 2. The activity of SOD was found to be decreased $33 \%$ and $37 \%$ in fluoride treated YEx and OEx treated rats when compared with their age matched controls YC and $\mathrm{OC}$ respectively, While, $35 \%$ decrease due to aging in OEx as compared with YEx. The activity of CAT was also markedly reduced by $34 \%$ and $65 \%$ in YEx and OEx as comparison with YC and OC respectively. The CAT reduced by $57 \%$ in OEx when compared with YEx. The glutathione containing enzyme GPx and GR were found to be significantly reduced by $22 \%$ and $17 \%$ in YEx, while $39 \%$ and 
$21 \%$ reduction in OEx as compared to their respective controls. Due to aging, GPx and GR were reduced by $34 \%$ and $29 \%$ in OEx as comparison with YEx.
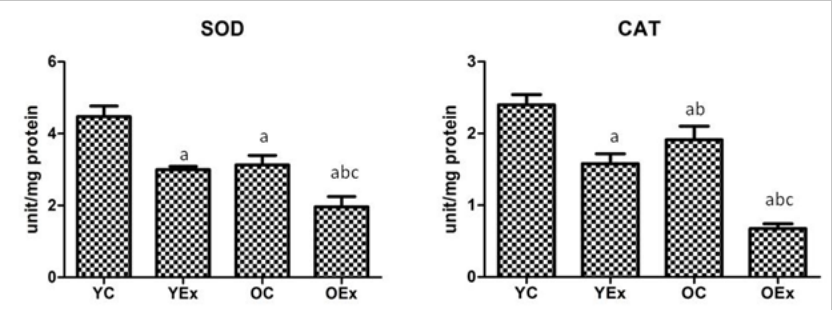

GR
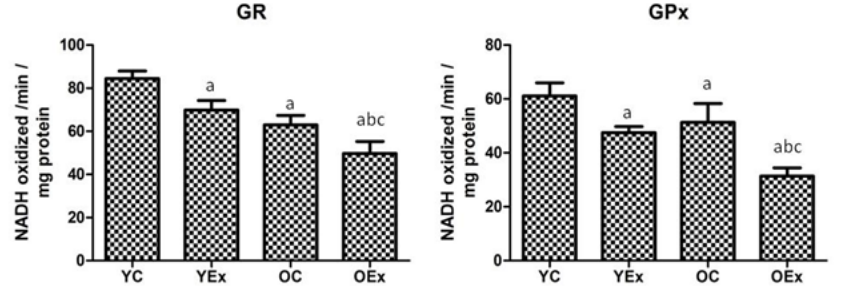

Figure 2 Activity of superoxide dismutase (SOD), Catalase (CAT), Glutathione peroxidase (GSHPx) and Glutathione reductase (GR) in control and fluoride treated group.The results are expressed as Mean \pm SEM in six rat of each group. Superscripts relate significant $(p<0.05)$ comparison with YC (a), YEx (b) and OC (c).

The concentration of GSG, GSSG and their ration are presented in Figure 3. The concentration of GSH was significantly reduced by $28 \%$ and $43 \%$ as compared to YC and OC respectively. The GSH was reduced by $32 \%$ in OEx in comparison to YEx. On the other hand, the concentration of GSSG was found to markedly elevate by $63 \%$ and $34 \%$ in YEx and OEx when compared with YC and OC respectively. OEx exhibited 30\% increment of GSSG as compared with the YEx. The GSH/GSSG ratio were markedly decreased by $56 \%$ in YEx and $58 \%$ in OEx when compared with their age matched controls respectively, while ratio was reduced by $48 \%$ in OEx as comparison with the YEx.

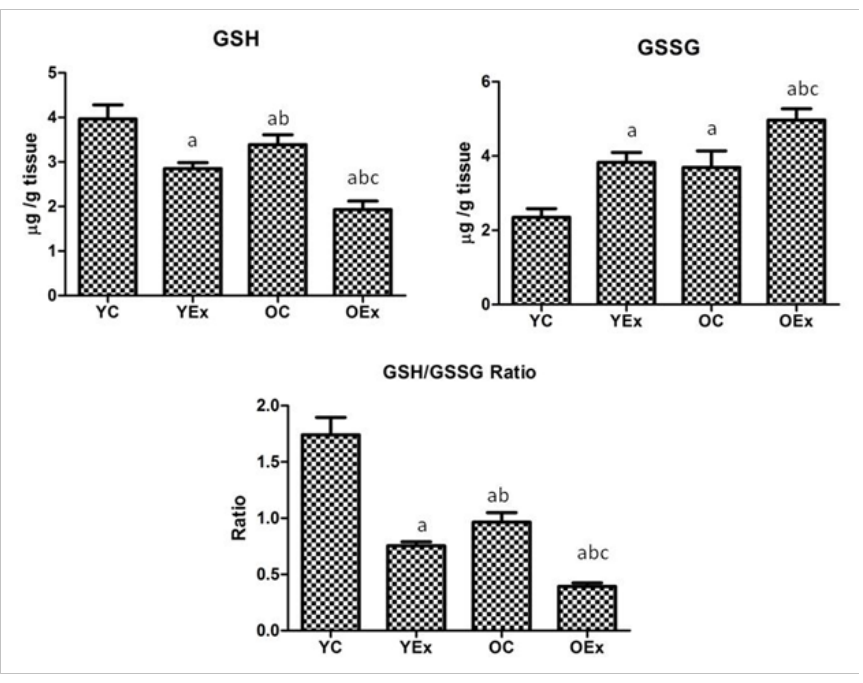

Figure 3 Levels of reduced glutathione (GSH), oxidized glutathione (GSSG) and their ratio (GSH/GSSG) in control and fluoride treated group. The results are expressed as Mean \pm SEM in six rat of each group. Superscripts relate significant $(p<0.05)$ comparison with YC (a), YEx (b) and OC (c).

Fluoride administration is known to cause oxidative modification of proteins, lipids and $\mathrm{DNA}^{24}$ and increased lipofuscin concentration could deleteriously affect the neurons, leading to depletion of antioxidants status..$^{25}$ The brain is known to possess many antioxidant enzymes; among them SOD plays a major role as it prevents ROS mediated tissue damage. ${ }^{26}$ Glutathione and catalase are important antioxidants, in the brain which protect it from $\mathrm{H}_{2} \mathrm{O}_{2}$ mediated neuronal damage and therefore decreased level of these biomolecules, ${ }^{27}$ as observed by us, may lead to increased severity of fluoride induced cerebral damage (Figure 2).

In the present study, we also found reduced a GSH/GSSG ratio in fluoride young and old rats (Figure 3). GSH/GSSG ratio determines the relative amount of reduced or working glutathione (GSH) compared to the oxidized glutathione (GSSG). A larger ratio reflects a more efficient glutathione redox system, because GSH is normally maintained in a highly reduced state of via NADPH dependent enzymes, specifically GR, while, GPx has protects from the $\mathrm{H}_{2} \mathrm{O}_{2}$ which produced during the metabolism of superoxide radicals. ${ }^{7}$ Our electron microscopic examination of the hippocampal neuron showed significant deposition of the lipofuscin granules with advancing age. Electron microscopy of both the young and aged rat (Figure 4A-D) brains revealed that neurons of the hippocampal region exhibited occasional small, round to irregular lipofuscin granules mingled with mitochondrial profiles in the immediate vicinity of the nuclei. The aged control rats had mostly irregular lipofuscin and in the fluoride treated aged rats (Figure 4C \& 4D). Moreover, the number and size of granules remarkably increased in fluoride exposed aged rats. Increasing intra-neuronal accumulation of lipofuscin is the most consistent cytological change, which has been correlated with fluoride neurotoxicity and ageing. It may be noted here that clustered lipofuscin granules were also found in the fluoride treated aged rats. Although, the increased content of lipofuscin in fluoride exposed young rats may be the hallmark of natural aging (Figure 4). Furthermore, changes observed in fluoride exposed young rats resembled those of old control animals which indicate that fluoride is responsible for premature aging of animals. Lipofuscin in itself is a cytotoxic agent which may induce a variety of ultra structural changes, like cell vacuolization, mitochondrial swelling and demylination in brain tissue. ${ }^{28}$ Hence elevated fluoride content along with high neuro lipofuscin levels may possibly play a role in the development of neurodegenerative disorders.

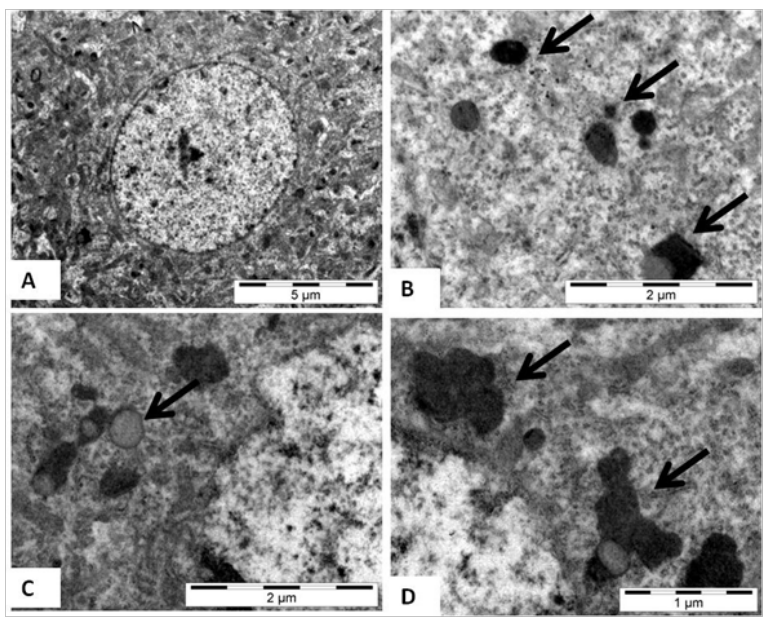

Figure 4 Electron micrograph of young control has shown a nucleus along with a nucleolus of hippocampus from a young rat. Part of perikaryon is also, which is rich in electron dense bodies and mitochondrial profiles (A). Fluoride intoxicated young rat (B) exhibited a number of variegated lipofuscin (arrow) pigment granules. EM of part of a perikaryon from the hippocampus of old control rat showing pleomorphic lipofuscin granules mingled with a few lysosomes (C). EM of old experimental rat showing a peripheral segment of perikaryon of a neuron with large number of pleomorphic irregular clustered lipofuscin granules (D). 


\section{Conclusion}

In the present study, aged rats were more prone to fluoride induced lipid peroxidation, increased lipofuscinogenesis and reduced antioxidant level. The biochemical and ultrastrural changes observed in fluoride treated young rats were comparable to those of untreated old control rats. Therefore, fluoride may play a role in the premature aging of animals. However, further in depth studies in other models are required to unravel the role of fluoride in the pathophysiology of aging.

\section{Acknowledgements}

Author wish to thank NIMS University for providing infrastructure for the research.

\section{Conflict of interest}

The author declares no conflict of interest.

\section{References}

1. Reddy GB, Khandare AL, Reddy PY, et al. Antioxidant Defense System and Lipid Peroxidation in Patients with Skeletal Fluorosis and in Fluoride-Intoxicated Rabbits. Toxicol Sci. 2003;72(2):363-368.

2. Fluoride in Drinking-water. Background document for development of WHO Guidelines for Drinking-water Quality. 2004.

3. Lou DD, Guan ZZ, Liu YJ, et al. The influence of chronic fluorosis on mitochondrial dynamics morphology and distribution in cortical neurons of the rat brain. Arch Toxicol. 2013;87(3):449-457.

4. DenBesten PK, Thariani H. Biological Mechanisms of Fluorosis and Level and Timing of Systemic Exposure to Fluoride with Respect to Fluorosis. J Dent Res. 1992;71(5):1238-1243.

5. Sarkar C, Pal S, Das N, et al. Ameliorative effects of oleanolic acid on fluoride induced metabolic and oxidative dysfunctions in rat brain: Experimental and biochemical studies. Food Chem Toxicol. 2014;66:224-236.

6. Oteiza PL, Keen CL, Han B, et al. Aluminum accumulation and neurotoxicity in swiss-webster mice after long-term dietary exposue to aluminum and citrate. Metabolism. 1993;42(10):1296-1300.

7. Tripathi S, Mahdi AA, Nawab A, et al. Influence of age on aluminum induced lipid peroxidation and neurolipofuscin in frontal cortex of rat brain: A behavioral, biochemical and ultrastructural study. Brain Research. 2009;1253:107-116.

8. Atmaca N, Atmaca HT, Kanici A, et al. Protective effect of resveratrol on sodium fluoride-induced oxidative stress, hepatotoxicity and neurotoxicity in rats. Food Chem Toxicol. 2014;70:191-197.

9. Montoliu C, Valles S, Renau-Piqueras J, et al. Ethanol-induced oxygen radical formation and lipid peroxidation in rat brain:effect of chronic alcohol consumption. J Neurochem. 1994;63(5):1855-1862.

10. Lowry OH, Rosebrough NJ, Farr AL, et al. Protein measurement with the Folin phenol reagent. J Biol Chem. 1951;193(1):265-275.

11. Liu R, Liu IY, Thompson RF, et al. Reversal of age related learning and brain oxidative stress in mice with superoxide dismutase/catalase mimetics. Proc Natl Acad Sci USA. 2003;100(14):8526-8531.
12. Ohkawa H, Ohishi N, Yagi K. Assay for lipid peroxides in animal tissue by thio-barbituric acid reaction. Anal Biochem. 1979;95(2):351-358.

13. Haldebrandt AG, Roots I. Reduced nicotinamide adenine dinucleotide phosphate (NADPH) dependent formation and breakdown of hydrogen peroxide during mixed function oxidation reaction in liver microsome. Arch Biochem Biophys. 1975;171(2):385-397.

14. Racknagel RD, Ghosal AK. Quantitative estimation of peroxidative degeneration of rat liver microsomal and mitochondrial lipids alters carbon tetrachloride poisoning. Exp Mol Pathol. 1966;5(5):413-426.

15. Sohal RS, Donato H. Effect of experimenal prolongation of life span on lipofuscin content and lysosomal enzyme activity in the brain of the housefly, Musca domestica. J Gerontol. 1979;34(4):489-496.

16. McCord JM, Fridovich I. SOD enzyme function for erythrocuprein. $J$ Biol Chem. 1969;224(22):6049-6055.

17. Aebi H. Catalase. In: Bergmeyer HU, editor. Methods of Enzymatic Analysis. New York: Academic Press Inc; 1974. p. 673-684.

18. Paglia DE, Valentine WN. Studies on the quantitative and qualitative characterization of erythrocyte glutathione peroxidase. Lab Clin Med. 1967;70(1):158-169.

19. Hazelton GA, Lang CA. Glutathione peroxidase and reductase activities in the aging mouse. Mech Ageing Dev. 1985;29(1):71-81.

20. Ellman GL. Tissue sulfydryl groups. Arch Biochem Biophysics. 1959;82(1):70-77.

21. Folbergrova J, Rehncron S, Siesj BK. Oxidized and reduced glutathione in the rat brain under normoxic and hypoxic conditions. $J$ Neurochem. 1979;32(6):1621-1627.

22. Sauerheber R. Physiologic Conditions Affect Toxicity of Ingested Industrial Fluoride. Journal of Environmental and Public Health. 2013;2013:439490.

23. Jesberger JA, Richardson JS. Oxygen free radicals and brain dysfunction. Int $J$ Neurosci. 1991;57(1-2):1-17.

24. Fatma EA, Mohamed O El-Badry, Hassan Dina AA, et al. Role of Vitamin E in Combination with Methionine and L- carnosine Against Sodium Fluoride-Induced Hematological, Biochemical, DNA Damage, Histological and Immunohistochemical Changes in Pancreas of Albino Rats. Life Science Journal. 2012;9(2):1260-1275.

25. Suh JH, Moreau R, Heath SH, et al. Dietary supplementation with (R)- $\alpha$-lipoic acid reverses the age-related accumulation of iron and depletion of antioxidants in the rat cerebral cortex. Redox Report. 2005;10(1):52-60.

26. Mates JM. Effects of antioxidant enzymes in the molecular control of reactive oxygen species toxicology. Toxicology. 2000;153(1-3):83-104

27. Bains JS, Shaw CA. Neurodegenerative disorders in humans: the role of glutathione in oxidative stress-mediated neuronal death. Brain Research Reviews. 1997;25(3):335-358.

28. Saad El-Dien HM, El Gamal DA, Mubarak HA, et al. Effect of Fluoride on Rat Cerebellar Cortex: Light and Electron Microscopic Studies. Egypt J Histol. 2010;33(2):245-256. 